Original Article (short paper)

\title{
Who are the Brazilian adolescents most actives during commuting to school? a population-based study
}

\author{
Raphael Henrique de Oliveira Araujo ${ }^{1}$ (D) , Danilo Rodrigues Pereira da Silva ${ }^{1}$ (D) , Thayse Natacha Queiroz Ferreira \\ Gomes $^{1}$ (i) , Antônio Evaldo dos Santos ${ }^{1}$ (i) , Josiene de Oliveira Couto ${ }^{1}$ [D , Roberto Jerônimo dos Santos Silva ${ }^{1}$ (C) \\ ${ }^{1}$ Universidade Federal de Sergipe, Departamento de Educação Física, São Cristóvão, SE, Brasil
}

\begin{abstract}
Aims: 1) to identify the prevalence of active commuting to school (ACS) among Brazilian regions; and 2) to determine associated factors related to ACS in this population. Methods: Cross-sectional study comprising 16,493 adolescents (mean age 14.09 \pm 2.12 years). The data comes from the National School Health Survey (2015), and the information was collected by a self-reported questionnaire. Logistic regression models were performed to identify correlates of ACS. Results: Adolescents who live in the Southeast are more prone to have ACS compared to those who live in other regions. Do not have motor vehicles been positively associated with ACS [girls: 2.04 (1.72;2.42); boys $1.85(1.63 ; 2.10)]$. Those whom self-reported white was less prone to have ACS compared to their peers from other ethnicities. Those enrolled in private schools [girls: $0.43(0.34 ; 0.54)$; boys $(0.45(0.39 ; 0.53)$ ] and schools setting in rural area [girls: $0.38(0.25 ; 0.57)$; boys: $0.51(0.37 ; 71)$ ] are less prone to show ACS. In addition, adolescents who accumulated less active time during physical education classes [girls: $0.80(0.66 ; 0.97)$ ] and extracurricular shifts [boys: $0.69(0.60 ; 0.80)]$ were less prone to have an ACS, compared to their most active peers. Lastly, girls who spent $\leq 2$ hours presented fewer odds to have an ACS [0.75(0.63;0.90)]. Conclusions: ACS was most prevalent among those who live in the Southeast region and seems to be negativity associated with the socioeconomic level. Moreover, less active adolescents during both school and leisure time may be more prone to have passive travel go/from school.
\end{abstract}

Keywords: adolescents, motor activity, National Adolescent School-based Health Survey

\section{Introduction}

The World Health Organization (WHO) recommends that adolescents should be engaged in at least $60 \mathrm{~min} /$ day of moderate-to-vigorous physical activity, to improve health and prevent diseases ${ }^{1}$. This recommendation can be expressed as a result of the sum of activities performed in different domains, such as work/school, transport, household chores, and leisure time) $)^{2}$.

Despite the benefits of physical activity for health, children and adolescents worldwide do not reach the international recommendation $^{3}$, highlighting the need to formulate effective strategies for active lifestyle promotion in the different domains of the day, given that lower levels of physical activity are associated with poor mental health ${ }^{4}$ and the development of non-communicable disease $^{5,6}$. Thus, to contribute to the achievement of physical activity guidelines, it has been recommended that children and adolescents have active commuting to/from school (ACS), such as walking and/or cycling ${ }^{7,8}$.

Besides, researches carried out worldwide have sought to estimate the prevalence of adolescents who go/from schools in an active way ${ }^{3}$. In developing countries, like Brazil, it is estimated that about 34.4 to $73 \%$ of adolescents go/come from school by an active way ${ }^{9}$, being that this variation may be influenced by different methods used to assess ACS. Further, data about active transport in Brazil have been based majority in studies carried out in South and Southeast regions, while that data from the Northeast region is still little explored; lastly, information from North and Midwest regions were few reported by the previous literature? .

Despite ACS may be associated with health benefits ${ }^{10}$, evidence has shown a decline in the prevalence of adolescents who are active in their travels to/from school ${ }^{11}$. Thus, the scientific community has sought to identify possible barriers and facilitators of ACS, where, potential levels of influence on active transport have been highlighted, such as intrapersonal characteristics (e.g. sex, age), social (e.g. family income), and environmental factors (e.g. distance to school, urbanization) $)^{12}$. Although of earlier efforts, some information about the association between "independent variables" and "ACS" is still not clear when founds from different countries are contrasted. For example, in Colombia ${ }^{13}$ and Mexico ${ }^{14}$, adolescents who live in rural areas are more prone to be active in their travels to school, while the opposite seems to be true in Canada ${ }^{15}$ and $\mathrm{USA}^{16}$.

The scientific community also have alerted that although the majority of studies are carried out in most developed countries, it needs to be careful to use these finds to guide police markers in low- and middle-income countries, given that this may ignore costs of successful interventions, and applicability or relevance specific to a context where the studies were carried ${ }^{17}$. Lastly, most of the studies carried out in Brazil sought to identify the 
correlates of ACS among adolescents considering a local's sample ${ }^{18,19}$, but not the entire population. Therefore, as far as we are aware, only a study ${ }^{20}$, using data from 2012, considered a cross-national sample. Thus, since identifying the correlates of ACS can help to guide public policies across the country, mainly to groups most exposed to insufficient physical activity, our purpose was to identify the prevalence and correlates of ACS among Brazilian adolescents.

\section{Methods}

In the present study, we used data from the 2015 National School Health Survey (in Portuguese: Pesquisa Nacional de Saúde do Escolar-PeNSE), which were collected between Abril and September 2015 by Brazilian Institute of Geography and Statistics, Ministry of Health and Ministry of Education. The PeNSE is cross-national school-based research, conducted every three years, since 2009, and its main objective was to provide information about risk and protection factors in students enrolled in public and private schools in the five macro-regions of the country.

Briefly, PeNSE 2015 database is composed of students from the 6th year of elementary school to the 3rd grade of high school, in the reference year of the research. The initial sample was included 16556 students; however, due to missing data about ACS, 63 subjects were excluded. Thus, our analyses are based on 16,493 adolescents, aged $11-19$ years (mean age $14.09 \pm 2.12$ years, $50.8 \%$ boys). The sample size was calculated for each stratum and considered a sampling error of $3 \%$, prevalence of $50 \%$, with a confidence interval of $95 \%$. Data were collected through a self-administered electronic questionnaire, composed of two main sections. The first related to the school characteristics were filled out by the principal or coordinator. The second section was self-administered by students and involved individual characteristics.

Based on the 2013 school census, data collection were processed taking into account schools, that were selected according to geographic strata (North, Northeast; Southeast; Midwest, South), sampling by conglomerates; schools from each Brazilian Major Region were selected, and in each school's classes were selected according to inclusion criteria; from these selected classes, all students were invited to take part in the study, and only those who answered the questionnaire were included in the study, totalizing 653 classes from 371 schools. More details regarding sampling strategies can be accessed in the original PeNSE methods document21. The PeNSE 2015 was approved by the National Commission for Ethics in Research from the National Health Council (Protocol No. 1.006.467).

\section{Variables in the study}

\section{Active commuting to and from school}

To assess ACS, two questions were used: 1) "In the last 7 days, how many days did you go on foot or cycle to school?"; 2)
"In the last 7 days, how many days did you get back on foot or by bike from school?". Hence, the adolescents who reported "to/ from" school $\geq 5$ times per week were classified as "active" 22 .

\section{Sociodemographic variables}

We assessed the following sociodemographic variables: age; ethnicity (white; black; yellow; brown; and indigenous); Country Region (North; Northeast; Southeast; South; Midwest); maternal education level (incomplete elementary school, elementary school; high school; and undergraduate); the presence of a motorized vehicle in a household (none or at least 1). We also used information about the type of school (private or public) and school setting (rural or urban) to assess information about the school environment. Hence, the perception of safety on the way to school was measured through the question "In the last 30 days, how many days did you miss school because you didn't feel safe on the way home from school or from school to home?", the students who related "miss school" at least once during this time were considered as those who "not safe".

\section{Body mass index (BMI)}

The BMI was calculated through weight $(\mathrm{kg})$ divided by height squared (m). To statistical analyses, the students were sorted in "normal weight" or "overweight", where we adopted the cut-offs suggested by $\mathrm{WHO}^{23}$.

\section{Behavioural variables}

The physical activity during physical education classes was estimated through the following questions: a) "How many days did you take PE classes at school?"; b) "How much time per day did you do physical activity or sport during physical education classes at school?", these questions were multiplied, and its result was computed in minutes per week. Hence the extracurricular physical activity was estimated by a) "In the last 7 days, except for school physical education classes, how many days did you engage in any physical activity, such as sports, dancing, gymnastics, bodybuilding, wrestling or other activity?"; b) "Usually, how long per day do these activities (such as sports, dance, gymnastics, bodybuilding, wrestling, or other activity) do you do? (Not counting physical education classes)", these questions were multiplied, and its result was computed in minutes per week. To statistical analyse, both PA indicators were categorized in tertile ( $\mathrm{min} /$ week), according to sex [physical activity during physical education: female: $(0 ; 1$ to $50 ;>50)$; and male: $(0$ to $15 ;>15$ to $70 ;>70)$ ] and [extracurricular physical activity: female: $(0 ;>0$ to $70 ;>70)$; and male ( 0 to $30 ;>30$ to $195 ;>195)$ ].

Lastly, sitting time was assessed by the question: "On a typical weekday, how long do you sit around watching television, using a computer, playing video games, talking to friends, or doing other sitting activities? (do not count Saturday, Sunday, 
holidays and time sitting at school)"; sitting time was sorted in " $\leq 2$ hours/day", " $>2$ to $\leq 4$ hours a day", and " $>4$ hours/ day".

\section{Data analysis}

Descriptive analyses were performed by absolute and relative frequencies according to the sex. The logistic regression models (crude and adjusted) was used to identify the variables associated with the ACS, with a $95 \%$ confidence interval. The variables with $\mathrm{p} \leq 0.2$ in the crude analysis were included in the adjusted model. In the adjusted model, the following hierarchical approach was adopted to variables inclusion: Model 1 (Individual characteristics): age; ethnicity; BMI. Model 2 (geographical characteristics), including Model 1 plus country region. Model 3 (socioeconomic variables), including model 2 plus educational level; private vehicle; Model 4 (school characteristics), including model 3 plus school type; school setting. Model 5 (perceived environment), including model 4 plus "perception of safety on the way to school". Model 6 (behavioural variables), including Model 5 plus "physical activity during physical education classes"; "extracurricular physical activity" and sitting time. All statistical analyses were performed in SPSS ${ }^{\circledR} 22$ (IBM, Armonk, New York, USA), with the statistical significance level of $\mathrm{p} \leq 0.05$.

\section{Results}

Table 1 shows the sample descriptive characteristics by sex. Most of the sample were brown, normal weight, from the urban setting, had at least one motor vehicle in their home, enrolled in public school, and reported good perception of safe on way to school.

Moreover, Figure 1 presents the prevalence of ACS in Brazil and each Brazilian region, by sex. Also, our analyses revealed that the ACS was not different among boys and girls adjustments [boys vs girls: Odds Ratio $=1.07(0.98 ; 1.17)]$, not even when covariates (individual, geographical, socioeconomic and perceived environment) were included [boys vs girls: Odds Ratio $=1.08(0.97 ; 1.20)$ ]

The crude analysis is shown in Table 2 , where the variables that showed " $p<0,20$ " were used in the adjusted analysis. Hence, in the final statistic model, (Table 3 and Table 4) we observed that to live in the Southeast region and "have not" a motor vehicle been associated with ACS. On the other hand, those whom self-reported "white" presented less prone to have ACS. Likewise, those enrolled in private schools and schools setting in the rural area are less prone to show ACS. Regarding variables related to physical activity, girls who accumulated less active time during physical education classes and boys who accumulated less active time during extracurricular shifts were less prone to have an ACS, compared to most active peers. We also observed that girls who spent less sitting time showed less probability to go/come from schools in an active way.

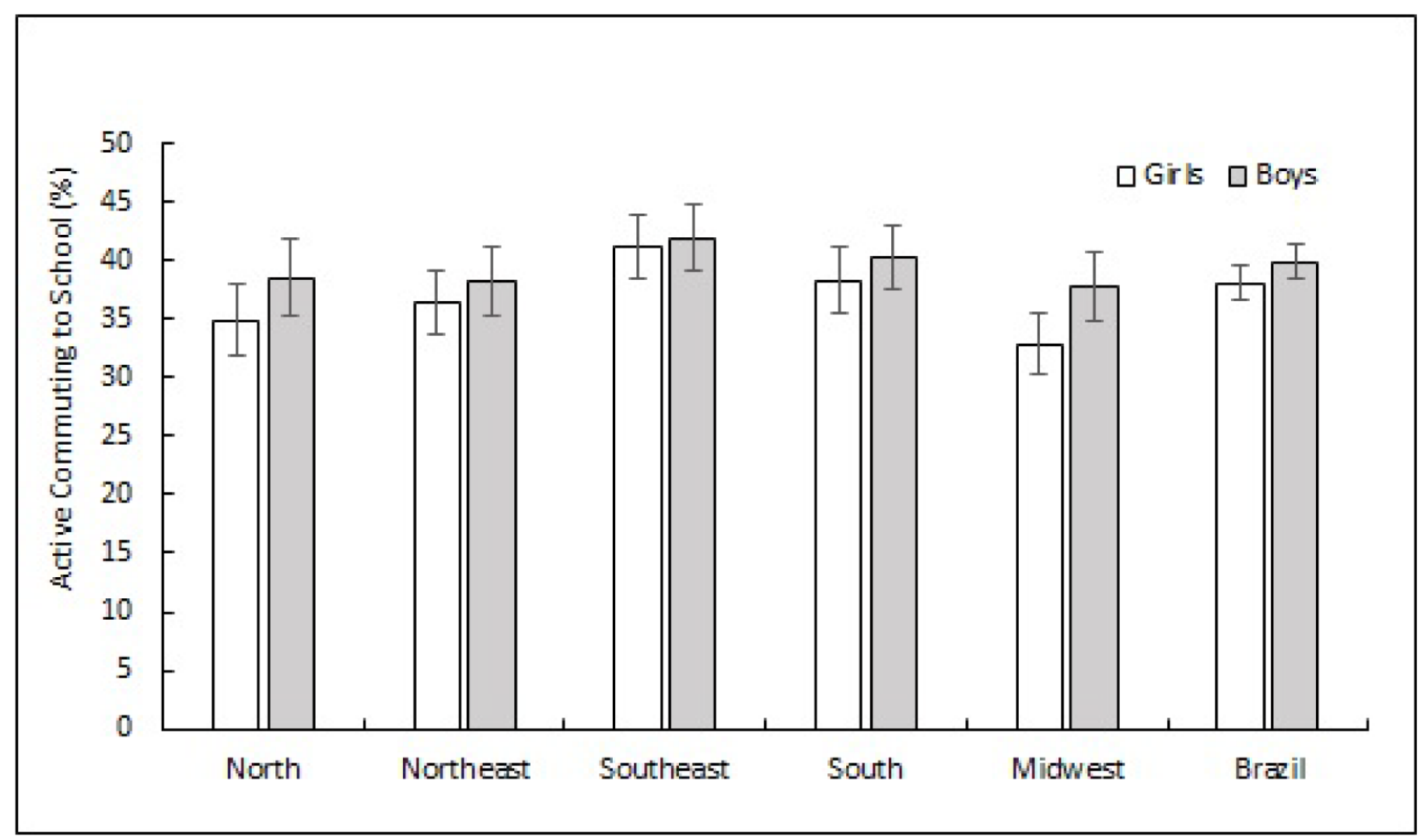

Figure 1- Prevalence of active commuting to school according to geographical region and sex. 
Table 1- Sample characteristics by sex $(n=16,493)$.

\begin{tabular}{|c|c|c|}
\hline \multirow{2}{*}{ Variables } & Female & Male \\
\hline & $\%(95 C I)$ & $\%(95 \mathrm{CI})$ \\
\hline \multicolumn{3}{|l|}{ Ethnicity } \\
\hline White & $35.4(34.0 ; 36.8)$ & $37.6(36.1 ; 39.9)$ \\
\hline Black & $10.9(10.0 ; 11.9)$ & $15.6(14.5 ; 16.9)$ \\
\hline Yellow & $4.7(4.1 ; 5.3)$ & $3.7(3.1 ; 4.3)$ \\
\hline Brown & $46.1(44.6 ; 47.6)$ & $39.7(38.2 ; 41.2)$ \\
\hline Indigenous & $2.9(2.5 ; 3.5)$ & $3.4(2.9 ; 4.0)$ \\
\hline \multicolumn{3}{|l|}{ BMI } \\
\hline Normal & $74.1(72.7 ; 75.3)$ & $75.5(74.1 ; 76.7)$ \\
\hline Overweight & $25.9(24.7 ; 27.3)$ & $24.5(23.3 ; 25.9)$ \\
\hline \multicolumn{3}{|l|}{ Region } \\
\hline North & $9.7(9.2 ; 10.2)$ & $9.5(9.0 ; 10.0)$ \\
\hline Northeast & $28.8(27.7 ; 29.8)$ & $30.2(29.0 ; 31.4)$ \\
\hline Southeast & $40.8(39.8 ; 42.1)$ & $39.3(38.2 ; 40.6)$ \\
\hline South & $12.8(12.3 ; 13.3)$ & $13.5(12.9 ; 14.0)$ \\
\hline Midwest & $7.9(7.5 ; 8.2)$ & $7.5(7.1 ; 7.9)$ \\
\hline \multicolumn{3}{|l|}{ Maternal educational level } \\
\hline Incomplete elementary school & $36.1(34.4 ; 37.8)$ & $33.1(31.4 ; 34.9)$ \\
\hline Elementary school & $16.6(15.4 ; 18.0)$ & $18.4(17.0 ; 19.8)$ \\
\hline High school & $30.0(28.4 ; 31.6)$ & $29.9(28.3 ; 31.5)$ \\
\hline Undergraduate & $17.3(16.2 ; 18.5)$ & $18.6(17.4 ; 19.9)$ \\
\hline \multicolumn{3}{|l|}{ Motor vehicle } \\
\hline None & $33.0(31.6 ; 34.4)$ & $29.6(28.2 ; 31.0)$ \\
\hline At least 1 & $67.0(65.6 ; 68.4)$ & $70.4(69.0 ; 71.8)$ \\
\hline \multicolumn{3}{|l|}{ School type } \\
\hline Private & $14.3(13.6 ; 14.9)$ & $12.7(12.0 ; 13.3)$ \\
\hline Public & $85.7(85.1 ; 86.4)$ & $87.3(86.7 ; 88.0)$ \\
\hline \multicolumn{3}{|l|}{ School setting } \\
\hline Rural & $5.9(5.2 ; 6.6)$ & $6.8(6.0 ; 7.7)$ \\
\hline Urban & $94.1(93.4 ; 94.8)$ & $93.2(92.3 ; 94.0)$ \\
\hline \multicolumn{3}{|l|}{ Perception of safety on the way to school } \\
\hline Not safe & $13.1(12.1 ; 14.1)$ & $14.5(13.4 ; 15.7)$ \\
\hline Safe & $86.9(85.9 ; 87.9)$ & $85.5(84.3 ; 86.6)$ \\
\hline \multicolumn{3}{|l|}{ Physical activity in school } \\
\hline Tertile 1 & $41.0(39.5 ; 42.4)$ & $42.1(40.6 ; 43.7)$ \\
\hline Tertile 2 & $30.4(29.0 ; 31.8)$ & $32.6(31.2 ; 34.0)$ \\
\hline Tertile 3 & $28.6(27.4 ; 30.0)$ & $25.3(24.1 ; 26.3)$ \\
\hline \multicolumn{3}{|l|}{ Physical activity out of school } \\
\hline Tertile 1 & $47.2(45.7 ; 48.7)$ & $37.3(35.9 ; 38.9)$ \\
\hline Tertile 2 & $24.6(23.4 ; 25.9)$ & $34.0(32.5 ; 35.4)$ \\
\hline Tertile 3 & $28.2(26.9 ; 29.5)$ & $28.7(27.3 ; 30.0)$ \\
\hline \multicolumn{3}{|l|}{ Sitting time } \\
\hline$\leq 2$ hours & $36.5(35.1 ; 38.0)$ & $38.2(36.7 ; 39.7)$ \\
\hline$>2$ to $\leq 4$ hours & $22.8(21.6 ; 24.1)$ & $25.2(23.9 ; 26.5)$ \\
\hline$>4$ hours & $40.7(39.2 ; 42.2)$ & $36.6(35.2 ; 38.1)$ \\
\hline
\end{tabular}


Table 2- Crude logistic regression for correlates of active commuting to schools among Brazilians adolescents.

\begin{tabular}{|c|c|c|c|c|}
\hline \multirow{2}{*}{ Variables } & \multicolumn{2}{|c|}{ Female } & \multicolumn{2}{|c|}{ Male } \\
\hline & OR $(95 \%$ CI) & p & OR (95\% CI) & p \\
\hline Age: & $1.00(0.97 ; 1.03)$ & 0.557 & $1.03(1.00 ; 1.06)$ & 0.051 \\
\hline \multicolumn{5}{|l|}{ Ethnicity $\dagger$ t: } \\
\hline Indigenous & $1.17(0.81 ; 1.70)$ & 0.385 & $0.99(0.69 ; 1.42)$ & 0.989 \\
\hline Black & $1.19(0.96 ; 1.47)$ & 0.111 & $1.40(1.15 ; 1.71)$ & 0.001 \\
\hline Yellow & $1.29(0.96 ; 1.74)$ & 0.083 & $1.54(1.09 ; 2.17)$ & 0.013 \\
\hline Brown & $1.17(1.02 ; 1.35)$ & 0.025 & $1.19(1.03 ; 1.37)$ & 0.016 \\
\hline White & 1 & & 1 & \\
\hline \multicolumn{5}{|l|}{ BMI } \\
\hline Overweight & 1 & & 1 & \\
\hline Normal & $0.86(0.85 ; 1.13)$ & 0.868 & $1.00(0.86 ; 1.16)$ & 0.945 \\
\hline \multicolumn{5}{|l|}{ Region†t: } \\
\hline North & $0.76(0.64 ; 0.78)$ & 0.003 & $0.86(0.72 ; 1.03)$ & 0.118 \\
\hline Northeast & $0.82(0.69 ; 0.96)$ & 0.018 & $0.85(0.72 ; 1.01)$ & 0.074 \\
\hline Midwest & $0.70(0.59 ; 0.82)$ & $<0.001$ & $0.84(0.71 ; 0.99)$ & 0.041 \\
\hline South & $0.89(0.75 ; 1.04)$ & 0.161 & $0.93(0.79 ; 1.09)$ & 0.391 \\
\hline Southeast & 1 & & 1 & \\
\hline \multicolumn{5}{|l|}{ Maternal educational level $\uparrow *$} \\
\hline Incomplete elementary & $2.01(1.61 ; 2.50)$ & $<0.001$ & $1.63(1.31 ; 2.02)$ & $<0.001$ \\
\hline Elementary school & $1.97(1.54 ; 2.53)$ & $<0.001$ & $2.00(1.58 ; 2.53)$ & $<0.001$ \\
\hline High school & $1.89(1.51 ; 2.36)$ & $<0.001$ & $1.86(1.51 ; 2.29)$ & $<0.001$ \\
\hline Undergraduate & 1 & & 1 & \\
\hline \multicolumn{5}{|l|}{ Motor vehicle $\uparrow$ t } \\
\hline At least 1 & 1 & & 1 & \\
\hline None & $1.92(1.68 ; 2.20)$ & $<0.001$ & $1.69(1.47 ; 1.95)$ & $<0.001$ \\
\hline \multicolumn{5}{|l|}{ School typet: } \\
\hline Public & 1 & & 1 & \\
\hline Private & $0.38(0.31 ; 0.46)$ & $<0.001$ & $0.41(0.34 ; 0.48)$ & $<0.001$ \\
\hline \multicolumn{5}{|l|}{ School setting $\dagger:$} \\
\hline Urban & 1 & & 1 & \\
\hline Rural & $0.40(0.29 ; 0.56)$ & $<0.001$ & $0.64(0.45 ; 0.91)$ & 0.015 \\
\hline \multicolumn{5}{|c|}{ Perception of safety on the way to school: } \\
\hline Safe & 1 & & 1 & \\
\hline Not safe & $1.07(0.89 ; 1.29)$ & 0.465 & $0.72(0.59 ; 0.88)$ & 0.001 \\
\hline \multicolumn{5}{|l|}{ Physical activity in school $\dagger$} \\
\hline Tertile 1 & $0.93(0.80 ; 1.08)$ & 0.352 & $0.91(0.74 ; 1.06)$ & 0.242 \\
\hline Tertile 2 & $0.78(0.67 ; 0.91)$ & 0.002 & $0.94(0.80 ; 1.10)$ & 0.474 \\
\hline Tertile 3 & 1 & & 1 & \\
\hline \multicolumn{5}{|l|}{ Physical activity out school $\$$} \\
\hline Tertile 1 & $1.09(0.94 ; 1.27)$ & 0.205 & $0.66(0.57 ; 0.78)$ & 0.000 \\
\hline Tertile 2 & $1.03(0.88 ; 1.22)$ & 0.658 & $0.81(0.69 ; 0.95)$ & 0.010 \\
\hline Tertile 3 & 1 & & 1 & \\
\hline \multicolumn{5}{|l|}{ Sitting time $\uparrow:$} \\
\hline$\leq 2$ hours & $0.76(0.65 ; 0.88)$ & 0.000 & $0.86(0.74 ; 1.00)$ & 0.057 \\
\hline$>2$ to $\leq 4$ hours & $0.93(0.79 ; 1.09)$ & 0.396 & $0.91(0.77 ; 1.07)$ & 0.279 \\
\hline$>4$ hours & 1 & & 1 & \\
\hline
\end{tabular}

Note: variables included in the adjusted model for girlst and boys $\neq . O R=$ Odds Ratio. 
Table 3 - Adjusted logistic models for correlates of active commuting to schools in girls.

\begin{tabular}{|c|c|c|c|c|c|c|c|c|c|c|c|c|}
\hline \multirow{2}{*}{ Variables } & \multicolumn{2}{|c|}{ Model 1} & \multicolumn{2}{|c|}{ Model 2} & \multicolumn{2}{|c|}{ Model 3} & \multicolumn{2}{|c|}{ Model 4} & \multicolumn{2}{|c|}{ Model 5} & \multicolumn{2}{|c|}{ Model 6} \\
\hline & OR (95\% CI) & $p$ & OR (95\% CI) & $p$ & OR $(95 \%$ CI $)$ & $p$ & OR $(95 \% \mathrm{CI})$ & $p$ & OR $(95 \% \mathrm{CI})$ & $p$ & OR $(95 \% \mathrm{CI})$ & $p$ \\
\hline Age & - & & - & & - & & - & & - & & - & \\
\hline \multicolumn{13}{|l|}{ Ethnicity } \\
\hline Indigenous & $\begin{array}{c}1.17(0.81 \\
1.70)\end{array}$ & 0.385 & $\begin{array}{c}1.24(0.86 \\
1.79)\end{array}$ & 0.245 & $\begin{array}{c}1.09(0.69 ; \\
1.71)\end{array}$ & 0.69 & $\begin{array}{c}1.09(0.69 ; \\
1.73)\end{array}$ & 0.693 & $\begin{array}{c}1.09(0.69 ; \\
1.73)\end{array}$ & 0.693 & $\begin{array}{c}1.10(0.69 ; \\
1.77)\end{array}$ & 0.665 \\
\hline Black & $\begin{array}{c}1.19(0.96 \\
1.47)\end{array}$ & 0.111 & $\begin{array}{c}1.22(0.98 ; \\
1.52)\end{array}$ & 0.065 & $\begin{array}{c}1.09(0.84 \\
1.43)\end{array}$ & 0.493 & $\begin{array}{c}1.05(0.80 \\
1.38)\end{array}$ & 0.700 & $\begin{array}{c}1.05(0.80 \\
1.38)\end{array}$ & 0.700 & $\begin{array}{c}1.07(0.82 \\
1.40)\end{array}$ & 0.607 \\
\hline Yellow & $\begin{array}{c}1.29(0.96 \\
1.74)\end{array}$ & 0.083 & $\begin{array}{c}1.35(1.00 \\
1.82)\end{array}$ & 0.047 & $\begin{array}{c}1.33(0.93 ; \\
1.91)\end{array}$ & 0.114 & $\begin{array}{c}1.27(0.88 \\
1.83)\end{array}$ & 0.19 & $\begin{array}{c}1.27(0.88 \\
1.83)\end{array}$ & 0.19 & $\begin{array}{c}1.25(0.87 ; \\
1.80)\end{array}$ & 0.211 \\
\hline Brown & $\begin{array}{c}1.17(1.02 \\
1.35)\end{array}$ & 0.025 & $\begin{array}{c}1.22(1.05 ; \\
1.42)\end{array}$ & 0.007 & $\begin{array}{c}1.03(0.86 ; \\
1.22)\end{array}$ & 0.713 & $\begin{array}{c}0.99(0.83 ; \\
1.18)\end{array}$ & 0.917 & $\begin{array}{c}0.99(0.83 ; \\
1.18)\end{array}$ & 0.917 & $\begin{array}{c}1.01(0.84 \\
1.20)\end{array}$ & 0.892 \\
\hline White & 1 & & 1 & & 1 & & 1 & & 1 & & 1 & \\
\hline BMI & - & & - & & - & & - & & - & & - & \\
\hline \multicolumn{13}{|l|}{ Region } \\
\hline North & & & $\begin{array}{l}0.74(0.62 ; \\
\quad 0.88)\end{array}$ & 0.001 & $\begin{array}{l}0.68(0.55 \\
0.85)\end{array}$ & 0.001 & $\begin{array}{c}0.69(0.55 \\
0.87)\end{array}$ & 0.002 & $\begin{array}{c}0.69(0.55 \\
0.87)\end{array}$ & 0.002 & $\begin{array}{l}0.71(0.57 \\
0.90)\end{array}$ & 0.005 \\
\hline Northeast & & & $\begin{array}{c}0.79(0.67 ; \\
0.93)\end{array}$ & 0.007 & $\begin{array}{l}0.74(0.61 ; \\
0.91)\end{array}$ & 0.004 & $\begin{array}{c}0.78(0.64 \\
0.95)\end{array}$ & 0.015 & $\begin{array}{l}0.78(0.64 \\
0.95)\end{array}$ & 0.015 & $\begin{array}{l}0.79(0.65 \\
0.97)\end{array}$ & 0.027 \\
\hline Midwest & & & $\begin{array}{l}0.68(0.58 \\
0.81)\end{array}$ & $<0.001$ & $\begin{array}{l}0.72(0.59 \\
0.88)\end{array}$ & 0.001 & $\begin{array}{c}0.72(0.59 \\
0.88)\end{array}$ & 0.002 & $\begin{array}{l}0.72(0.59 \\
0.88)\end{array}$ & 0.002 & $\begin{array}{c}0.73(0.59 \\
0.90)\end{array}$ & 0.003 \\
\hline South & & & $\begin{array}{c}0.93(0.79 \\
1.10)\end{array}$ & 0.423 & $\begin{array}{c}0.88(0.72 \\
1.08)\end{array}$ & 0.243 & $\begin{array}{l}0.82(0.67 ; \\
1.01)\end{array}$ & 0.069 & $\begin{array}{c}0.82(0.67 \\
1.01)\end{array}$ & 0.069 & $\begin{array}{c}0.82(0.67 \\
1.01)\end{array}$ & 0.069 \\
\hline Southeast & & & 1 & & 1 & & 1 & & 1 & & 1 & \\
\hline \multicolumn{13}{|l|}{$\begin{array}{c}\text { Maternal } \\
\text { educational } \\
\text { level }\end{array}$} \\
\hline $\begin{array}{l}\text { Incomplete } \\
\text { elementary }\end{array}$ & & & & & $\begin{array}{c}1.61(1.28 ; \\
2.02)\end{array}$ & $<0.001$ & $\begin{array}{c}1.23(0.96 ; \\
1.58)\end{array}$ & 0.088 & $\begin{array}{c}1.23(0.96 ; \\
1.58)\end{array}$ & 0.088 & $\begin{array}{c}1.29(1.01 ; \\
1.66)\end{array}$ & 0.039 \\
\hline $\begin{array}{c}\text { Elementary } \\
\text { school }\end{array}$ & & & & & $\begin{array}{c}1.67(1.29 ; \\
2.16)\end{array}$ & $<0.001$ & $\begin{array}{c}1.27(0.96 ; \\
1.66)\end{array}$ & 0.084 & $\begin{array}{c}1.27(0.96 ; \\
1.66)\end{array}$ & 0.084 & $\begin{array}{c}1.30(0.99 ; \\
1.71)\end{array}$ & 0.058 \\
\hline High school & & & & & $\begin{array}{l}1.63(1.31 \\
2.04)\end{array}$ & $<0.001$ & $\begin{array}{c}2.01(1.69 ; \\
2.38)\end{array}$ & $<0.001$ & $\begin{array}{l}2.01(1.69 ; \\
2.38)\end{array}$ & $<0.001$ & $\begin{array}{c}1.33(1.05 \\
1.69)\end{array}$ & 0.018 \\
\hline Undergraduate & & & & & 1 & & 1 & & 1 & & 1 & \\
\hline \multicolumn{13}{|l|}{ Motor vehicle } \\
\hline At least 1 & & & & & 1 & & 1 & & 1 & & 1 & \\
\hline None & & & & & $\begin{array}{l}2.15(1.82 \\
2.54)\end{array}$ & $<0.001$ & $\begin{array}{l}2.01(1.69 ; \\
2.38)\end{array}$ & $<0.001$ & $\begin{array}{l}2.01(1.69 ; \\
2.38)\end{array}$ & $<0.001$ & $\begin{array}{l}2.04(1.72 \\
2.42)\end{array}$ & $<0.001$ \\
\hline \multicolumn{13}{|l|}{ School type } \\
\hline Public & & & & & & & 1 & & 1 & & 1 & \\
\hline Private & & & & & & & $\begin{array}{c}0.45(0.36 \\
0.56)\end{array}$ & $<0.001$ & $\begin{array}{c}0.45(0.36 \\
0.56)\end{array}$ & $<0.001$ & $\begin{array}{c}0.43(0.34 \\
0.54)\end{array}$ & $<0.001$ \\
\hline \multicolumn{13}{|l|}{ School setting } \\
\hline Urban & & & & & & & 1 & & 1 & & 1 & \\
\hline Rural & & & & & & & $\begin{array}{l}0.36(0.24 \\
0.53)\end{array}$ & $<0.001$ & $\begin{array}{l}0.36(0.24 \\
0.53)\end{array}$ & $<0.001$ & $\begin{array}{l}0.38(0.25 \\
0.57)\end{array}$ & $<0.001$ \\
\hline \multicolumn{13}{|l|}{$\begin{array}{l}\text { way to school } \\
\text { Physical activ- } \\
\text { ity in school }\end{array}$} \\
\hline Tertile 1 & & & & & & & & & & & $\begin{array}{l}0.80(0.66 \\
0.97)\end{array}$ & 0.023 \\
\hline Tertile 2 & & & & & & & & & & & $\begin{array}{c}0.69(0.57 \\
0.84)\end{array}$ & $<0.001$ \\
\hline Tertile 3 & & & & & & & & & & & 1 & \\
\hline $\begin{array}{l}\text { Physical activ- } \\
\text { ity out school }\end{array}$ & & & & & & & & & & & - & \\
\hline \multicolumn{13}{|l|}{ Sitting time } \\
\hline$\leq 2$ hours & & & & & & & & & & & $\begin{array}{l}0.75(0.63 \\
0.90)\end{array}$ & $<0.001$ \\
\hline $\begin{array}{c}>2 \text { to } \leq 4 \\
\text { hours }\end{array}$ & & & & & & & & & & & $\begin{array}{l}0.95(0.78 \\
1.15)\end{array}$ & 0.621 \\
\hline$>4$ hours & & & & & & & & & & & 1 & \\
\hline
\end{tabular}

Note: Adjusted by: Model 1: age; ethnicity; BMI. Model 2: including Model 1 plus country region. Model 3: including model 2 plus maternal educational level; private vehicle; Model 4: including model 3 plus school type; school setting. Model 5: including model 4 plus "perception of safety on the way to school". Model 6: including Model 5 plus "physical activity during physical education classes"; "extracurricular physical activity" and sitting time. The variables that did not obtain a significant value in crude analyses $(\mathrm{p}<0.20)$ were not included in adjusted analyses. OR = Odds Ratio. 
Table 4 - Adjusted logistic models for correlates of active commuting to schools in boys.

\begin{tabular}{|c|c|c|c|c|c|c|c|c|c|c|c|c|}
\hline \multirow{2}{*}{ Variables } & \multicolumn{2}{|c|}{ Model 1} & \multicolumn{2}{|c|}{ Model 2} & \multicolumn{2}{|c|}{ Model 3} & \multicolumn{2}{|c|}{ Model 4} & \multicolumn{2}{|c|}{ Model 5} & \multicolumn{2}{|c|}{ Model 6} \\
\hline & OR $(95 \% \mathrm{CI})$ & $p$ & OR $(95 \% \mathrm{CI})$ & $p$ & OR $(95 \%$ CI $)$ & $p$ & OR $(95 \% \mathrm{CI})$ & $p$ & OR $(95 \% \mathrm{CI})$ & $p$ & OR $(95 \% \mathrm{CI})$ & $p$ \\
\hline Age & $\begin{array}{c}1.02(0.99 \\
1.04)\end{array}$ & 0.083 & $\begin{array}{c}1.02(1.00 \\
1.06)\end{array}$ & 0.053 & $\begin{array}{c}1.01(0.99 ; \\
1.04)\end{array}$ & 0.185 & $\begin{array}{c}1.00(0.97 ; \\
1.02)\end{array}$ & 0.891 & $\begin{array}{c}1.00(0.97 ; \\
1.02)\end{array}$ & 0.448 & $\begin{array}{c}1.00(0.97 ; \\
1.03)\end{array}$ & 0.805 \\
\hline \multicolumn{13}{|l|}{ Ethnicity } \\
\hline Indigenous & $\begin{array}{c}1.08(0.83 ; \\
1.39)\end{array}$ & 0.538 & $\begin{array}{c}1.25(0.87 ; \\
1.45)\end{array}$ & 0.368 & $\begin{array}{c}1.11(0.81 ; \\
1.52)\end{array}$ & 0.498 & $\begin{array}{c}1.07(0.78 ; \\
1.47)\end{array}$ & 0.654 & $\begin{array}{c}1.08(0.79 ; \\
1.48)\end{array}$ & 0.616 & $\begin{array}{c}1.06(0.77 \\
1.46)\end{array}$ & 0.708 \\
\hline Black & $\begin{array}{c}1.31(1.13 ; \\
1.52)\end{array}$ & $<0.001$ & $\begin{array}{l}1.35(1.16 ; \\
1.56)\end{array}$ & $<0.001$ & $\begin{array}{c}1.25(1.04 ; \\
1.51)\end{array}$ & 0.006 & $\begin{array}{c}1.21(1.00 \\
1.46)\end{array}$ & 0.043 & $\begin{array}{c}1.23(1.01 \\
1.48)\end{array}$ & 0.031 & $\begin{array}{c}1.22(1.01 \\
1.47)\end{array}$ & 0.037 \\
\hline Yellow & $\begin{array}{c}1.40(1.12 \\
1.75)\end{array}$ & 0.003 & $\begin{array}{c}1.44(1.15 ; \\
1.81)\end{array}$ & 0.001 & $\begin{array}{c}1.45 \\
(1.11 ; 1.90)\end{array}$ & 0.006 & $\begin{array}{c}1.38(1.05 ; \\
1.82)\end{array}$ & 0.018 & $\begin{array}{c}1.40(1.06 \\
1.83)\end{array}$ & 0.015 & $\begin{array}{c}1.39(1.05 \\
1.83)\end{array}$ & 0.019 \\
\hline Brown & $\begin{array}{c}1.17(1.06 \\
1.30)\end{array}$ & 0.002 & $\begin{array}{c}1.21(1.09 ; \\
1.35)\end{array}$ & $<0.001$ & $\begin{array}{c}1.15(1.01 \\
1.30)\end{array}$ & 0.026 & $\begin{array}{c}1.09(0.96 \\
1.24)\end{array}$ & 0.149 & $\begin{array}{c}1.09(0.96 ; \\
1.24)\end{array}$ & 0.154 & $\begin{array}{c}1.11(0.98 \\
1.26)\end{array}$ & 0.098 \\
\hline White & 1 & & 1 & & 1 & & 1 & & 1 & & 1 & \\
\hline BMI & - & & - & & - & & - & & - & & - & \\
\hline \multicolumn{13}{|l|}{ Region } \\
\hline North & & & $\begin{array}{l}0.79(0.70 ; \\
0.89)\end{array}$ & $<0.001$ & $\begin{array}{c}0.75(0.65 ; \\
0.88)\end{array}$ & $<0.001$ & $\begin{array}{c}0.77(0.66 ; \\
0.90)\end{array}$ & 0.002 & $\begin{array}{c}0.77(0.66 ; \\
0.90)\end{array}$ & 0.002 & $\begin{array}{c}0.78(0.66 ; \\
0.91)\end{array}$ & 0.002 \\
\hline Northeast & & & $\begin{array}{l}0.81(0.71 \\
0.91)\end{array}$ & 0.001 & $\begin{array}{c}0.73(0.63 \\
0.84)\end{array}$ & $<0.001$ & $\begin{array}{c}0.76(0.66 \\
0.88)\end{array}$ & $<0.001$ & $\begin{array}{c}0.76(0.66 \\
0.88)\end{array}$ & $<0.001$ & $\begin{array}{c}0.77(0.67 \\
0.89)\end{array}$ & $<0.001$ \\
\hline Midwest & & & $\begin{array}{l}0.76(0.67 \\
0.85)\end{array}$ & $<0.001$ & $\begin{array}{c}0.80(0.69 ; \\
0.92)\end{array}$ & 0.002 & $\begin{array}{c}0.81(0.70 \\
0.94)\end{array}$ & 0.006 & $\begin{array}{c}0.81(0.70 \\
0.93)\end{array}$ & 0.004 & $\begin{array}{c}0.79(0.69 \\
0.92)\end{array}$ & 0.002 \\
\hline South & & & $\begin{array}{c}0.96(0.85 \\
1.07)\end{array}$ & 0.484 & $\begin{array}{c}0.97(0.84 ; \\
1.11)\end{array}$ & 0.67 & $\begin{array}{c}0.91(0.79 ; \\
1.05)\end{array}$ & 0.219 & $\begin{array}{c}0.91(0.79 \\
1.05)\end{array}$ & 0.198 & $\begin{array}{c}0.91(0.79 ; \\
1.05)\end{array}$ & 0.215 \\
\hline Southeast & & & 1 & & 1 & & 1 & & 1 & & 1 & \\
\hline & & & & & & & & & & & & \\
\hline $\begin{array}{l}\text { Incomplete } \\
\text { elementary }\end{array}$ & & & & & $\begin{array}{c}1.48(1.26 \\
1.74)\end{array}$ & $<0.001$ & $\begin{array}{c}1.21(1.02 \\
1.44)\end{array}$ & 0.024 & $\begin{array}{c}1.22(1.03 ; \\
1.45)\end{array}$ & 0.019 & $\begin{array}{c}1.27(1.08 \\
1.53)\end{array}$ & 0.004 \\
\hline $\begin{array}{l}\text { Elementary } \\
\text { school }\end{array}$ & & & & & $\begin{array}{l}1.72(1.45 ; \\
2.05)\end{array}$ & $\begin{array}{c}< \\
0.001\end{array}$ & $\begin{array}{c}1.39(1.16 \\
1.68)\end{array}$ & $<0.001$ & $\begin{array}{c}1.39(1.16 \\
1.68)\end{array}$ & $<0.001$ & $\begin{array}{c}1.43(1.19 \\
1.73)\end{array}$ & $<0.001$ \\
\hline High school & & & & & $\begin{array}{c}1.65(1.41 ; \\
1.92)\end{array}$ & $\begin{array}{c}< \\
0.001\end{array}$ & $\begin{array}{c}1.37(1.17 ; \\
1.61)\end{array}$ & $<0.001$ & $\begin{array}{c}1.37(1.17 ; \\
1.61)\end{array}$ & $<0.001$ & $\begin{array}{c}1.40(1.19 ; \\
1.64)\end{array}$ & $<0.001$ \\
\hline \multicolumn{13}{|l|}{ Motor vehicle } \\
\hline At least 1 & & & & & 1 & & 1 & & 1 & & 1 & \\
\hline None & & & & & $\begin{array}{c}1.91(1.70 ; \\
2.16)\end{array}$ & $<0.001$ & $\begin{array}{c}1.80(1.59 \\
2.04)\end{array}$ & $<0.001$ & $\begin{array}{c}1.81(1.59 ; \\
2.04)\end{array}$ & $<0.001$ & $\begin{array}{c}1.85(1.63 ; \\
2.10)\end{array}$ & $<0.001$ \\
\hline $\begin{array}{l}\text { School type } \\
\text { Public }\end{array}$ & & & & & & & 1 & & 1 & & 1 & \\
\hline Private & & & & & & & $\begin{array}{c}0.47(0.40 \\
0.55)\end{array}$ & $<0.001$ & $\begin{array}{c}0.47(0.40 ; \\
0.54)\end{array}$ & $<0.001$ & $\begin{array}{c}0.45(0.39 ; \\
0.53)\end{array}$ & $<0.001$ \\
\hline $\begin{array}{c}\text { School setting } \\
\text { Urban }\end{array}$ & & & & & & & - & & - & & 1 & \\
\hline Rural & & & & & & & $\begin{array}{c}0.49(0.35 \\
0.68)\end{array}$ & $<0.001$ & $\begin{array}{c}0.48(0.34 \\
0.67)\end{array}$ & $<0.001$ & $\begin{array}{c}0.51(0.37 \\
0.71)\end{array}$ & $<0.001$ \\
\hline \multicolumn{13}{|l|}{$\begin{array}{l}\text { Perception of } \\
\text { safety on the }\end{array}$} \\
\hline $\begin{array}{c}\text { Not safe } \\
\text { Physical activity }\end{array}$ & & & & & & & & & $\begin{array}{c}0.83(0.71 \\
0.98)\end{array}$ & 0.031 & $\begin{array}{c}0.83(0.71 \\
0.98)\end{array}$ & 0.029 \\
\hline \multicolumn{13}{|l|}{$\begin{array}{l}\text { Physical activity } \\
\text { in school } \\
\text { Physical activity } \\
\text { out school }\end{array}$} \\
\hline $\begin{array}{c}\text { out school } \\
\text { Tertile } 1\end{array}$ & & & & & & & & & & & $0.69(0.60$ & $<0.001$ \\
\hline Tertile 2 & & & & & & & & & & & $\begin{array}{c}0.78(0.67 \\
0.90)\end{array}$ & 0.001 \\
\hline Tertile 3 & & & & & & & & & & & 1 & \\
\hline \multicolumn{13}{|l|}{ Sitting time } \\
\hline$\leq 2$ hours & & & & & & & & & & & $\begin{array}{c}0.81(0.71 \\
0.92)\end{array}$ & 0.002 \\
\hline$>2$ to $\leq 4$ hours & & & & & & & & & & & $\begin{array}{c}0.95(0.83 \\
1.09)\end{array}$ & 0.471 \\
\hline$>4$ hours & & & & & & & & & & & 1 & \\
\hline
\end{tabular}

Note: Adjusted by: Model 1: age; ethnicity; BMI. Model 2: including Model 1 plus country region. Model 3: including model 2 plus maternal educational level; private vehicle; Model 4: including model 3 plus school type; school setting. Model 5: including model 4 plus "perception of safety on the way to school". Model 6: including Model 5 plus "physical activity during physical education classes"; "extracurricular physical activity" and sitting time. The variables that did not obtain a significant value in crude analyses $(p<0.20)$ were not included in adjusted analyses. OR = Odds Ratio. 


\section{Discussion}

This is a cross-sectional study carried with data from PeNSE 2015. Our purposes were 1) identify the prevalence of ACS among Brazilian regions, and; 2) to determine associated factors related to ACS in this population. Our results presented that those who live in the Southeast region have most odds to be active in their travels to schools when compared to adolescents who live in Northeast, North, and Midwest. Adolescents who related "have not a motor vehicle" in the household also were most prone to present ACS. On the other hand, those who self-reported "white" were less prone to have ACS compared to those from other ethnicities. Besides, adolescents enrolled in private schools and schools setting in rural areas are less prone to show ACS. Girls less active during physical education classes and boys less active during extracurricular shifts were less prone to have an ACS, compared to most active peers. Lastly, girls who spent less sitting time showed less probability to go/from schools in an active way.

We did not observe differences between the patterns of commuting to schools of young and older adolescents. Besides, the pattern observed in the present study seems to be different from founds in other countries, like China ${ }^{11}$ and USA ${ }^{24}$. In the USA, for example, the passive travel to school among most old adolescents may be explained due to those with age upper to 16 years may getting their "drive license", while that in Brazil minimum age is 18 years. Moreover, an early study ${ }^{25}$ carried out in Brazil suggested that older adolescents may be most likely to have an ACS due to more independent to go/from school by walking or cycling compared to their peers most young. Thus, the association between age and ACS seems to be inconsistent and might range according to local's characteristics.

It was observed that ACS was most likely among adolescents who live in the Southeast region compared to those who live in the Northeast, North, and Midwest. These finds are inconsistent with a study carried out in Mexico ${ }^{14}$, where those who live in less developed regions were more prone to go/from school on foot or cycling. Moreover, although our study did not investigate direct measures, like distance of the home to schools, previous studies allow us to hypothesize that fewer numbers of schools localized in rural areas in Southeast ${ }^{26}$, as well as its highly demographic density compared to North, Northeast, and Midwest ${ }^{27}$ may to encourage the ACS. Likewise, our results suggest that urban adolescents tend to be engaged in more ACS than rural ones. In Brazil, students from rural areas usually use free public transport to go to/from school due to distance and access from their home to schools, leading them to use passive commuting in this way, in opposition to students from the urban area, who tend to study in schools near their houses ${ }^{28,29}$.

Regarding the perception of safety, it was verified that boys who did not feel safe on the way to school showed lower odds to be active in their traveling to school. In Brazil, subjects aged 15-29 years are those more prone to be dead by firearm, and male subjects represent $91 \%-96 \%$ of the victims ${ }^{30}$, reinforced by the fact that weapon carrying and involvement in fights are more prevalent in male ${ }^{31}$. Thus, the risk behaviours related to violence can lead boys to avoid walking or cycling to school.
In addition, although our results were not consistent with the association between gender and ACS, previous studies have been relating to the fact that parents may feel insecure about letting their daughters going alone to school, using an active mode, being more protective of girls than with boys ${ }^{7,32}$.

In the present study, we also sought to identify whether socioeconomic level may be correlated to ACS among Brazilian adolescents, and our data analyses revealed that "maternal educational level", "having motor vehicle" and "to be enrolled in private school" were negatively associated to ACS. These finds are consistent with early studies carried out in China ${ }^{11}$ and Mexico $^{14}$. Thus, it is suggested that having at least one motor vehicle at home encourages its use rather than the use of active travel to school. It is important to highlight that individuals who use active transportation mode do not always do this for health or environmental awareness reasons, but maybe socioeconomic issues given the low costs related to walking or cycling comparing to an active transportation mode can influence this choice ${ }^{33}$.

Adolescents who are less active during physical education classes and extracurricular periods also are less prone to go/from school on foot or cycling, compared to their peers most actives (tertile 3). The relation between physical activity and ACS still yet not clear, and associations positives and negatives between both behaviours can be found in available literature ${ }^{11,34,35}$. On the other hand, our found are consistent with most of the studies presented previously by a systematic review ${ }^{35}$, suggesting that adolescents who present ACS are more prone to engage in other physical activity domains. Moreover, to identify that students who have passive commuting are groups most exposed to insufficient physical activity adds interesting information to guide public policies. Thus, it is plausible to suggest efforts to improve ACS among adolescents, mainly to those who less active in other physical activity domain, once that ACS can be integrated to student's daily routines ${ }^{36}$ and there is at least 200 schooldays/year, meaning that the benefits of active travel may be obtained during more than half of the year.

Our data analyses pointed that, in Brazil, the ACS may be a required behaviour, being related to socioeconomic conditions, and not only one choice based on health benefits, meaning that interventions focused on the promotion of ACS should offer to subjects the possibility of choosing among the types of travel. Besides, changing in traveling mode may not be feasible for those who live in rural areas or areas far from their schools, due to climatic and/or structural conditions; thus, interventions aiming to increase the active time during school time and leisure physical activity also be used as ways to help adolescents stay active.

\section{Limitations}

Notwithstanding the relevance of the finds, this study has some limitations that should be noted. Firstly, the PeNSE data are self-reported, which is prone to some biases in the information collected, especially those regarding ACS. However, this does not impair the data, given that studies carried worldwide have used questionnaires to obtain data about traveling to school ${ }^{37,38}$. Secondly, the lack of information related to the intensity of ACS 
(light, moderate or vigorous), which could help in the explanation of the role of ACS in the total weekly accumulation of moderate-to-vigorous physical activity. However, some strengths can be pointed, such as the use of environment variables, given that these factors are associated with sedentary lifestyle ${ }^{39}$; and the use of a large population-based sample.

\section{Conclusion}

It was observed that ACS was most prevalent among those who live in the Southeast region, and seems to be negativity associated with the socioeconomic level. Moreover, less active adolescents during both school and leisure time may be more prone to have passive travel go/from school.

\section{References}

1. World Health Organization. Global recommendations on physical activity for health. Geneva: World Health Organization; 2010. 58 p.

2. Bauman AE, Reis RS, Sallis JF, Wells JC, Loos RJ, Martin BW, et al. Correlates of physical activity: why are some people physically active and others not? Lancet. 2012;380(9838):258-71

3. Aubert S, Barnes JD, Abdeta C, Abi Nader P, Adeniyi AF, AguilarFarias N, et al. Global Matrix 3.0 Physical Activity Report Card Grades for Children and Youth: Results and Analysis From 49 Countries. J Phys Act Heal. 2018;15(S2):S251-73.

4. Rodriguez-Ayllon M, Cadenas-Sánchez C, Estévez-López F, Muñoz NE, Mora-Gonzalez J, Migueles JH, et al. Role of Physical Activity and Sedentary Behavior in the Mental Health of Preschoolers, Children and Adolescents: A Systematic Review and Meta-Analysis. Sport Med. 2019;(0123456789).

5. Lee EY, Yoon K-H. Epidemic obesity in children and adolescents: risk factors and prevention. Front Med. 2018;12(6):658-66.

6. Saluja K, Rawal T, Bassi S, Bhaumik S, Singh A, Park MH, et al. School environment assessment tools to address behavioural risk factors of non-communicable diseases: A scoping review. Prev Med Reports. 2018;10(47):1-8.

7. Huertas-Delgado FJ, Herrador-Colmenero M, Villa-González E, Aranda-Balboa MJ, Cáceres MV, Mandic S, et al. Parental perceptions of barriers to active commuting to school in Spanish children and adolescents. Eur J Public Health. 2017;27(3):416-21.

8. Pizarro AN, Schipperijn J, Andersen HB, Ribeiro JC, Mota J, Santos MP. Active commuting to school in Portuguese adolescents: Using PALMS to detect trips. J Transp Heal. 2016;3(3):297-304.

9. Ferrari GL de M, De Victo ER, Ferrari TK, Solé D. Active transportation to school for children and adolescents from Brazil: a systematic review. Brazilian J Kinanthropometry Hum Perform. 2018 Aug 23;20(4):406-14.

10. Saunders LE, Green JM, Petticrew MP, Steinbach R, Roberts H. What Are the Health Benefits of Active Travel? A Systematic Review of Trials and Cohort Studies. Ruiz JR, editor. PLoS One. 2013 Aug 15;8(8):e69912.

11. Yang Y, Hong X, Gurney JG, Wang Y. Active Travel to and From School Among School-Age Children During 1997-2011 and Associated Factors in China. J Phys Act Heal. 2017 Sep;14(9):684-91.

12. Pinto A de A, Claumann GS, Angelo HCC de, Menezes EC, Dias DT, Pelegrini A. Active Commuting To School and Associated Factors Among Adolescents: a Systematic Review. J Phys Educ. 2017;28(1):1-11.

13. Arango CM, Parra DC, Eyler A, Sarmiento O, Mantilla SC, Gomez LF, et al. Walking or Bicycling to School and Weight Status among Adolescents From Montería, Colombia. J Phys Act Heal. 2016;8(s2):S171-7.

14. Jáuregui A, Medina C, Salvo D, Barquera S, Rivera-dommarco JA. Active Commuting to School in Mexican Adolescents : Evidence From the Mexican National Nutrition and Health Survey. J Phys Act Heal. 2014;12(8):1088-95.

15. Pabayo R, Gauvin L. Proportions of students who use various modes of transportation to and from school in a representative population-based sample of children and adolescents, 1999. Prev Med (Baltim). 2008 Jan;46(1):63-6.

16. Martin SL, Lee SM, Lowry R. National Prevalence and Correlates of Walking and Bicycling to School. Am J Prev Med. 2007 Aug;33(2):98-105.

17. Miranda JJ, Zaman MJ. Exporting" failure": why research from rich countries may not benefit the developing world. Rev Saude Publica. 2010;44(1):185-9.

18. Silva KS, Nahas M V, Borgatto AF, Oliveira ES, Del Duca GF, Lopes AS. Factors Associated With Active Commuting to School and to Work Among Brazilian Adolescents. J Phys Act Heal. 2011;8(7):926-33.

19. Becker L, Fermino R, Lima A, Rech C, Añez C, Reis R. Perceived barriers for active commuting to school among adolescents from Curitiba, Brazil. Rev Bras Atividade Física Saúde. 2017;22(1).

20. de Rezende LFM, Azeredo CM, Canella DS, Claro RM, de Castro IRR, Levy RB, et al. Sociodemographic and behavioral factors associated with physical activity in Brazilian adolescents. BMC Public Health. 2014;14(1):485.

21. Instituto Brasileiro de Geografia e Estatística (IBGE). Instituto Brasileiro de Geografia e Estatística, Pesquisa Nacional de Saúde do Escolar (PeNSE) 2015. IBGE. Rio de Janeiro; 2016. 256 p.

22. Werneck AO, Oyeyemi AL, Fernandes RA, Romanzini M, Ronque ERV, Cyrino ES, et al. Regional Socioeconomic Inequalities in Physical Activity and Sedentary Behavior Among Brazilian Adolescents. J Phys Act Heal. 2018 May 1;15(5):338-44.

23. de Onis M. Development of a WHO growth reference for school-aged children and adolescents. Bull World Health Organ. 2007;85(09):660-7.

24. McDonald NC, Brown AL, Marchetti LM, Pedroso MS. U.S. School Travel, 2009. Am J Prev Med. 2011; (2):146-51.

25. Santos CM, Júnior W, de Souza R, Barros SSH, Farias Júnior JC de, Barros MVG de. Prevalência e fatores associados à inatividade física nos deslocamentos para escola em adolescentes. Cad Saude Publica. 2010;26:1419-30.

26. Ministério da Educação, Instituto Nacional de Estudos e Pesquisas Educacionais Anísio Teixeira (INEP). Censo Escolar da Educação Básica 2016: notas estatísticas. Brasília: INEP; 2017. 28 p.

27. Instituto Brasileiro de Geografia e Estatística (IBGE). Densidade populacional: números e imagens [Internet]. [cited 2020 Jan 11]. Available from: https://mapasinterativos.ibge.gov.br/densidade/ 
28. Hutchinson J, White PCL, Graham H. Differences in the social patterning of active travel between urban and rural populations: findings from a large UK household survey. Int J Public Health. 2014;59(6):993-8.

29. Yang Y, Ivey SS, Levy MC, Royne MB, Klesges LM. Active Travel to School: Findings From the Survey of US Health Behavior in School-Aged Children, 2009-2010. J Sch Health. 2016;86(6):464-71.

30. Waiselfsz JJ. Mapa da violência 2016. Mapa da violência [Internet]. 2016;71. Available from: http://www.mapadaviolencia.org.br/ pdf2016/Mapa2016_armas_web.pdf

31. Santos AE dos, Araujo RH de O, Soares NMM, Lima J de O, Oliveira CC da C, Dantas EHM, et al. Health Risk Behavior Excels in High Socioeconomic Class Among Adolescents. Int J Dev Res. 2019;09(01):25305-9.

32. De Meester F, Van DD., De Bourdeaudhuij I, Cardon G. Parental perceived neighborhood attributes: associations with active transport and physical activity among 10-12 year old children and the mediating role of independent mobility. BMC Public Health. 2014;14:1-14.

33. Manyanga T, Makaza D, Mahachi C, Mlalazi TF, Masocha V, Makoni P, et al. Results From Zimbabwe's 2016 Report Card on Physical Activity for Children and Youth. J Phys Act Heal. 2016; Nov;13(s2):S337-42.

34. Mann M, Silver EJ, Stein REK. Active Commuting to School, Physical Activity, and Behavior Problems Among Third-Grade Children. J Sch Health. 2018;88(10):734-43.

35. Barranco-Ruiz Y, Guevara-Paz A, Ramírez-Vélez R, Chillón P, Villa-González E. Mode of Commuting to School and Its Association with Physical Activity and Sedentary Habits in Young Ecuadorian Students. Int J Environ Res Public Health. 2018;15(12):2704.

36. Buehler R, Pucher J, Bauman A. Physical activity from walking and cycling for daily travel in the United States, 2001-2017: Demographic, socioeconomic, and geographic variation. J Transp Heal. 2020;16:100811.
37. Østergaard L, Grøntved A, Børrestad LAB, Froberg K, Gravesen M, Andersen LB. Cycling to school is associated with lower BMI and lower odds of being overweight or obese in a large population-based study of Danish adolescents. J Phys Act Health. 2012;9(5):617-25.

38. Huang WY, Wong SH, He G. Is a Change to Active Travel to School an Important Source of Physical Activity for Chinese Children? Pediatr Exerc Sci. 2017;29(1):161-8.

39. Sallis JF, Cervero RB, Ascher W, Henderson KA, Kraft MK, Kerr J. An ecological approach to creating active living communities. Annu. Rev. Public Health. 2006;297-322.

\section{Acknowledgments}

This study was financed in part by the Coordenação de Aperfeiçoamento de Pessoal de Nível Superior - Brasil (CAPES) - Finance Code 001.

\section{Corresponding author}

Raphael Henrique de Oliveira Araujo

Department of Physical Education, Federal University of Sergipe, Marechal Rondon Avenue, São Cristóvão, Sergipe, Brazil, Zip code: 49100-000

Email: raphael.edfis@gmail.com

Editor: Romulo A Fernandes, UNESP/Presidente Prudente, SP, Brazil

Manuscript received on March 17, 2020

Manuscript accepted on June 12, 2020

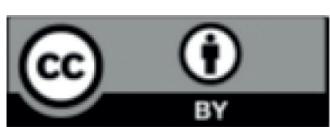

Motriz. The Journal of Physical Education. UNESP. Rio Claro, SP, Brazil - eISSN: 1980-6574 - under a license Creative Commons - Version 4.0 